\section{PREFACE VORWORT}

$\nearrow$ GERALD BAST // English translation: the editors

Art universities have always offered a working environment that avoids boundaries, encourages curiosity, and understands experimentation as a basic condition. Artistic research or arts-based research is nevertheless a comparatively new concept at international art universities and academies. There has been heated discussion over whether artistic research is even a legitimate term and whether research should not be reserved for the sciences.

The implementation of the FWF Programme for Arts-based Research (PEEK) has brought about a paradigm shift in the way artistic research is viewed and treated in terms of cultural policy. The program addresses the main conflict and problem areas within the discourse on artistic research and arrives at definitional statements of remarkable clarity and conciseness. "Every artistic production is based on the work and creativity of artists, who use artistic methods or skills to arrive at art-specific forms of expression. The Programme for Arts-based Research (PEEK) is about promoting that process in which artistic knowledge production is accompanied by reflection and thus leads to an increase in knowledge in society. [...] However, the creative process and its reception must be intersubjectively reflected upon, documented and presented in order to be sustainably available to artistic discourse and scientific research in the sense of arts-based research."

As in scientific research, the process of artistic research starts with the 'state of the art', it consciously places it at the beginning and not only presupposes it as a starting point, but also openly presents it as such. Artistic research is not only able to further develop the 'state of the art', but it is also able to cross disciplinary boundaries and to cooperate openly with other fields of science. Finally, artistic research is also essential towards the process of acquiring new artistic knowledge that is being documented and thus made comprehensible.
Seit jeher bieten Kunstuniversitäten ein Arbeitsumfeld, das Grenzen vermeidet, Neugier fördert und das Experimentieren als Grundbedingung begreift. Künstlerische Forschung oder Art-based Research ist dennoch ein vergleichsweise junges Kapitel an internationalen Kunstuniversitäten und -akademien. Die Diskussion, ob künstlerische Forschung überhaupt ein zulässiger Begriff und Forschung nicht für die Wissenschaften reserviert sei, wurde mitunter heftig geführt.

Die Installierung des FWF-Programms zur Entwicklung und Erschließung der Künste (PEEK) brachte einen kulturpolitisch paradigmatischen Perspektivenwechsel in der Sichtweise und der Behandlung künstlerischer Forschung. Das Programm greift die wesentlichen Konflikt- und Problemzonen des Diskurses über künstlerische Forschung auf und kommt zu definitorischen Feststellungen von bemerkenswerter Klarheit und Prägnanz. „Jede künstlerische Produktion basiert auf der Arbeit und der Kreativität von Künstler*innen, die mit künstlerischen Methoden bzw. Fertigkeiten zu kunstspezifischen Ausdrucksformen gelangen. Mit dem Programm zur Entwicklung und Erschließung der Künste (PEEK) geht es um die Förderung jenes Prozesses, bei dem künstlerische Wissensproduktion von Reflexion begleitet wird und so zu einem Erkenntnisgewinn in der Gesellschaft führt. [...] Der kreative Prozess und dessen Rezeption müssen jedoch intersubjektiv reflektiert, dokumentiert und präsentiert werden, um im Sinne der Art-based Research nachhaltig dem künstlerischen Diskurs und der wissenschaftlichen Forschung zur Verfügung zu stehen."1

Genau wie wissenschaftliche Forschung geht künstlerische Forschung in ihrem Prozess vom, state of the art' aus, sie stellt diesen bewusst an den Anfang und setzt ihn als Ausgangspunkt nicht nur voraus, sondern schildert ihn auch als solchen offen aus. Künstlerische Forschung vermag es nicht nur, den, state of the art' weiterzuentwickeln, sondern dabei auch disziplinäre Grenzen zu überschreiten und offen mit anderen Wissenschaftsfeldern zu kooperieren. Wesentlich ist schließlich auch, ob der Prozess zur Erlangung neuen künstlerischen Wissens dokumentiert und damit nachvollziehbar ist. 
The research project Conceptual Joining demonstrates all this in an impressive way. Christoph Kaltenbrunner's team combines engineering and scientific research with conceptual artistic techniques, thus creating interdisciplinary and, above all, new perspectives on cutting-edge questions. As a matter of course, they mix digital tools with traditional craft techniques, combining performance and architecture to create new worlds of experience.

I am particularly pleased that the results from this remarkable research project have now been collected in this publication. With this volume, the Angewandte is also making an important contribution towards shaping and defining artistic research.
Das Forschungsprojekt Conceptual Joining führt all das auf eindrucksvolle Art und Weise vor. Das Team rund um Christoph Kaltenbrunner verbindet Ingenieurswissenschaft und naturwissenschaftliche Forschung mit konzeptuellen künstlerischen Ansätzen und schafft so interdisziplinäre und vor allem neue Perspektiven auf hochaktuelle Fragen. Mit großer Selbstverständlichkeit mischen sie digitale Tools mit traditionellen Handwerkstechniken und kombinieren Performance und Architektur zu neuen Erfahrungswelten.

Es freut mich besonders, dass die Ergebnisse aus diesem bemerkenswerten Forschungsprojekt nun in dieser Publikation festgehalten werden. Mit dem vorliegenden Band leistet die Angewandte darüber hinaus einen wichtigen Beitrag zur Ausgestaltung und Definition künstlerischer Forschung.

1 en: Programme for Arts-based Research (PEEK), Program document FWF 2009, p. 3.

de: Programm zur Entwicklung und Erschließung der Künste (PEEK), Programmdokument FWF 2009, S. 3. 


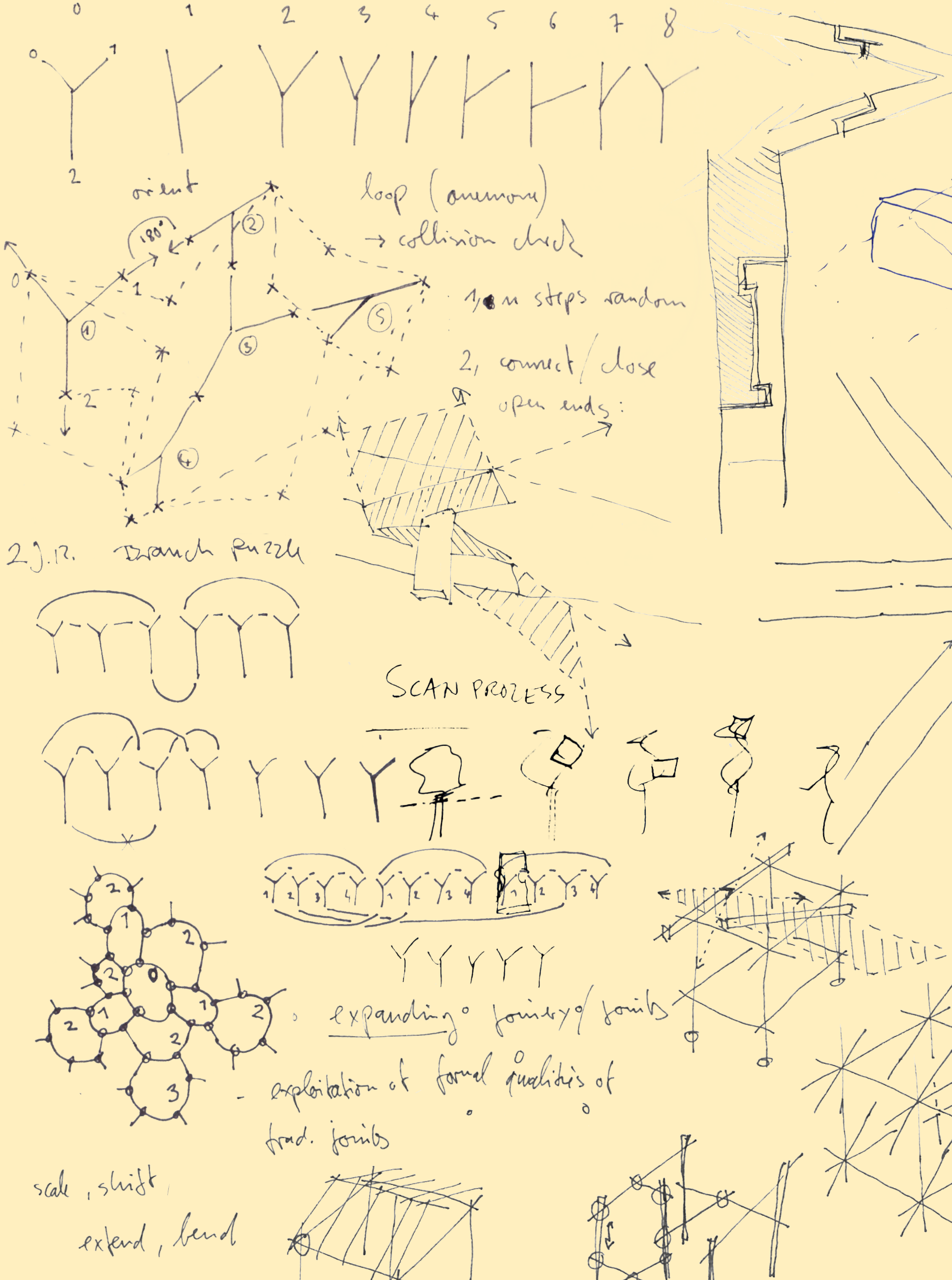




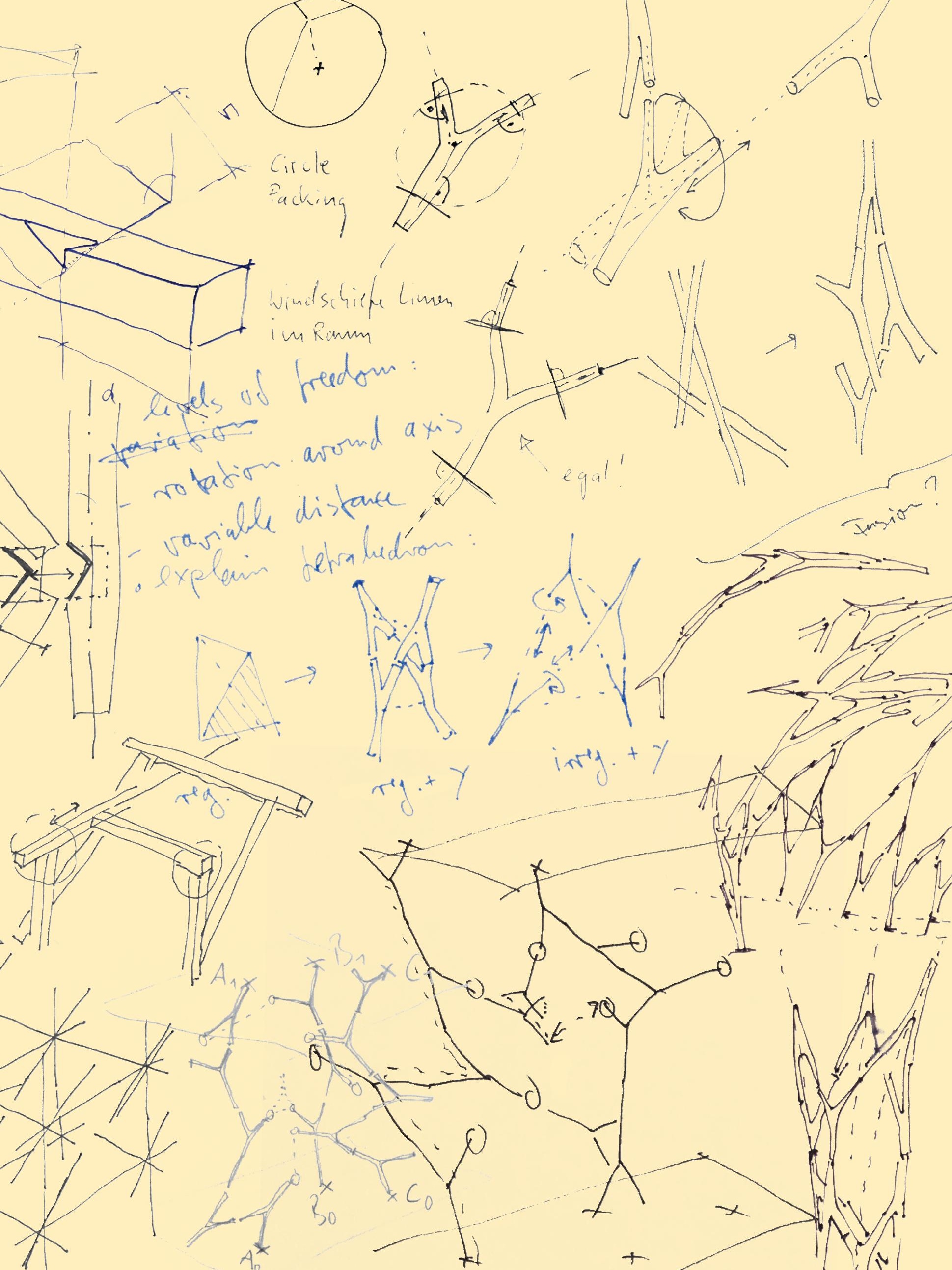

